\title{
A LIMIT THEOREM FOR STOCHASTIC NETWORKS AND ITS APPLICATIONS
}

\author{
E. O. LEBEDEV
}

\begin{abstract}
A service process in an overloaded regime for multichannel stochastic networks is considered. A general functional limit theorem is proved, and the properties of the limit process are studied. An application of the approximation obtained is given for the case of networks with a semi-Markov input.
\end{abstract}

\section{INTRODUCTION}

A multichannel network of queueing systems is the main model considered in the paper. Assume that customers arrive at an $i$ th node of the network, $i=1,2, \ldots, r$, at instances $\tau_{k}^{(i)}, k=1,2, \ldots$. Let $\nu_{i}(t)$ be the total number of customers arrived to the $i$ th node on the interval $[0, t]$. Every node consists of infinitely many similar servers. The service time of every server is exponentially distributed with parameter $\mu_{i}, i=1,2, \ldots, r$. After the service at an $i$ th node, a customer moves to a $j$ th node with probability $p_{i j}$, and exits the network with probability $p_{i r+1}=1-\sum_{j=1}^{r} p_{i j}$. Here $P=\left\|p_{i j}\right\|_{1}^{r}$ is the route matrix of the network. An extra, $(r+1)$ th, node is treated as the "exit" from the network. We denote this model by $[G|M| \infty]^{r}$.

The $[G|M| \infty]^{r}$ models of networks are used when designing computer or communication systems (see, for example, [1]) and in the studies of primary ionization processes (see [2]).

An $r$-dimensional process $Q(t)=\left(Q_{1}(t), \ldots, Q_{r}(t)\right)$ is called a service process in a $[G|M| \infty]^{r}$ network if $Q_{i}(t)$ is the number of busy servers at the $i$ th node at the moment $t \geq 0$. We study the service process $Q(t)$ for a critical traffic in the network. This means that the parameters of input flows $\nu_{i}(t)$ and service intensities $\mu_{i}, i=1,2, \ldots, r$, depend on " $n$ " (the series number), and moreover

1) there are constants $\lambda_{i}>0, i=1,2, \ldots, r$, for which

$$
n^{-1 / 2}\left(\nu_{1}^{(n)}(n t)-\lambda_{1} n t, \ldots, \nu_{r}^{(n)}(n t)-\lambda_{r} n t\right) \underset{n \rightarrow \infty}{\stackrel{U}{\Rightarrow}} W(t)=\left(W_{1}(t), \ldots, W_{r}(t)\right),
$$

where $W(t)$ is an $r$-dimensional Wiener process with zero mean vector,

$$
\mathrm{E} W(1)=0,
$$

and correlation matrix $\mathrm{E} W(1) W^{\prime}(1)=\sigma^{2}=\left\|\sigma_{i j}\right\|_{1}^{r}$. (The symbol $\stackrel{U}{\Rightarrow}$ stands for the weak convergence in the uniform topology);

2) $\lim _{n \rightarrow \infty} n \mu_{i}(n)=\mu_{i} \neq 0, i=1,2, \ldots, r$. 
E. O. LEBEDEV

Consider the sequence of stochastic processes

$$
\begin{gathered}
\xi^{(n)}(t)=n^{-1 / 2}\left(Q^{(n)}(n t)-n q(t)\right), \quad t \geq 0, \\
Q^{(n)^{\prime}}(0)=(0, \ldots, 0),
\end{gathered}
$$

where $q^{\prime}(t)=\left(q_{1}(t), \ldots, q_{r}(t)\right)=(\theta / \mu)^{\prime}(I-P(t)),(\theta / \mu)^{\prime}=\left(\theta_{1} / \mu_{1}, \ldots, \theta_{r} / \mu_{r}\right)$,

$$
\theta^{\prime}=\left(\theta_{1}, \ldots, \theta_{r}\right)=\lambda^{\prime}(I-P)^{-1}
$$

is a solution of the balance equation for a $[G|M| \infty]^{r}$ network, $\lambda^{\prime}=\left(\lambda_{1}, \ldots, \lambda_{r}\right)$,

$$
P(t)=\left\|p_{i j}(t)\right\|_{1}^{r}=\exp [\Delta(\mu)(P-I) t]
$$

and $\Delta(\mu)=\left\|\delta_{i j} \mu_{i}\right\|_{1}^{r}$ is a diagonal matrix.

The condition $Q^{(n) \prime}(0)=(0, \ldots, 0)$ means that the prelimit process is in a transient regime.

\section{The CONVERGENCE OF THE SERVICE PROCESS}

We introduce two independent Gaussian processes $\xi^{(1) \prime}(t)=\left(\xi_{1}^{(1)}(t), \ldots, \xi_{r}^{(1)}(t)\right)$ and $\xi^{(2) \prime}(t)=\left(\xi_{1}^{(2)}(t), \ldots, \xi_{r}^{(2)}(t)\right)$ in order to describe the limit behavior of the sequence $\xi^{(n)}(t), n \geq 1$.

The process $\xi^{1}(t)$ is completely determined by its mean value

$$
\mathrm{E} \xi^{(1)}(t)=0
$$

and correlation matrices

$$
\begin{gathered}
R^{(1)}(t)=\mathrm{E} \xi^{(1)}(t) \xi^{(1) \prime}(t)-\mathrm{E} \xi^{(1)}(t) \mathrm{E} \xi^{(1) \prime}(t)=\int_{0}^{t} P^{\prime}(u) \sigma^{2} P(u) d u, \\
R^{(1)}(s, t)=\mathrm{E} \xi^{(1)}(s) \xi^{(1) \prime}(t)-\mathrm{E} \xi^{(1)}(s) \mathrm{E} \xi^{(1) \prime}(t)=R^{(1)}(s) P(t-s), \quad s<t .
\end{gathered}
$$

The process $\xi^{(2)}(t)$ satisfies

$$
\begin{gathered}
\mathrm{E} \xi^{(2)}(t)=0, \\
R^{(2)}(t)=\sum_{m=1}^{r} \lambda_{m} \int_{0}^{t}\left(\Delta\left[p_{m}(u)\right]-p_{m}(u) p_{m}^{\prime}(u)\right) d u, \\
R^{(2)}(s, t)=R^{(2)}(s) P(t-s), \quad s<t,
\end{gathered}
$$

where $p_{m}^{\prime}(u)=\left(p_{m 1}(u), \ldots, p_{m r}(u)\right)$ is the $m$ th row of the matrix $P(u)$, and

$$
\Delta\left[p_{m}(u)\right]=\left\|p_{m i}(u) \delta_{i j}\right\|_{1}^{r}
$$

is a diagonal matrix.

The following theorem is the main result of the paper.

Theorem 1. Assume that a $\left[G^{(n)}\left|M^{(n)}\right| \infty\right]^{r}$ network of queue systems satisfies conditions 1) and 2). If the spectral radius of the route matrix $P$ is less than 1 , then the sequence of stochastic processes $\xi^{(n)}(t), n \geq 1$, converges to $\xi^{(1)}(t)+\xi^{(2)}(t)$ in the uniform topology on every finite interval $[0, T]$.

The proof of Theorem 1 is based on the following two auxiliary results.

Lemma 1. The finite-dimensional distributions of $\int_{0}^{t} d W^{\prime}(u) P(t-u)$ coincide with those of the Gaussian process $\xi^{(1)}(t)$. 
Lemma 1 follows from properties of the stochastic integral (see, for example, [3]).

The trajectory of a customer arrived at the network at an $m$ th node, can be described (until the time when it exits the network) by a Markov chain

$$
\eta^{(m)}(t) \in\{1,2, \ldots, r, r+1\}, \quad t \geq 0,
$$

whose infinitesimal matrix $\left\|q_{i j}\right\|_{1}^{r+1}$ and initial distribution $P\left(\eta^{(m)}(0)=i\right)$ are given by

$$
q_{i j}= \begin{cases}-\mu_{i}\left(1-p_{i i}\right), & i=j=1,2, \ldots, r, \\ \mu_{i} p_{i j}, & i \neq j, i=1,2, \ldots, r, j=1,2, \ldots, r, r+1, \\ 0, & i=r+1, j=1,2, \ldots, r, r+1,\end{cases}
$$

and $P\left(\eta^{(m)}(0)=i\right)=\delta_{m i}, i=1,2, \ldots, r+1$, respectively.

Let $\chi^{(m)}(t)=\left(\chi_{1}^{(m)}(t), \ldots, \chi_{r}^{(m)}(t)\right), t \geq 0, m=1, \ldots, r$, be an $r$-dimensional process defined by the chain $\eta^{(m)}(t)$ as follows:

$$
\chi^{(m)}= \begin{cases}e_{j}, & \eta^{(m)}(t)=j, j=1, \ldots, r, \\ e_{0}, & \eta^{(m)}(t)=r+1,\end{cases}
$$

where $e_{j}$ is an $r$-dimensional vector whose $j$ th coordinate is equal to 1 and all other coordinates are $0 ; e_{0}$ is the zero $r$-dimensional vector.

For an arbitrary positive integer $N$ and

$$
z^{\prime}(j)=\left(z_{1}(j), \ldots, z_{r}(j)\right), \quad j=1,2, \ldots, N,|z(j)| \leq 1,
$$

we denote by $\Phi^{(m)}=\Phi^{(m)}\left(t_{1}, \ldots, t_{N}, z(1), \ldots, z(N)\right)$ the joint moment generating function of the vectors $\chi^{(m)}\left(t_{1}\right), \ldots, \chi^{(m)}\left(t_{N}\right), 0<t_{1}<\cdots<t_{N}$,

$$
\Phi^{\prime}=\left(\Phi^{(1)}, \ldots, \Phi^{(r)}\right) \text {. }
$$

Lemma 2. For an arbitrary $N=1,2, \ldots$ and $0<t_{1}<\cdots<t_{N}$,

$$
\Phi=\overline{1}+\sum_{j=1}^{N} P\left(\Delta t_{1}\right) \Delta[z(1)] \cdots P\left(\Delta t_{j-1}\right) \Delta[z(j-1)] P\left(\Delta t_{j}\right)(z(j)-\overline{1}),
$$

where $\overline{1}$ is the $r$-dimensional vector whose coordinates are $1 s$, and $\Delta t_{i}=t_{i}-t_{i-1}\left(t_{0}=0\right)$ and $\Delta[z(i)]=\left\|z_{k}(i) \delta_{k m}\right\|_{1}^{r}$ are diagonal matrices.

Equality (1) can be proved by induction.

Proof of Theorem 1. There are two steps in the proof:

a) we prove the convergence of finite-dimensional distributions;

b) we show that

$$
\lim _{\Delta \rightarrow 0} \varlimsup_{n \rightarrow \infty} \mathrm{P}\left(\omega_{\Delta}\left(\xi^{(n)}\right)>\delta\right)=0
$$

for all $\delta>0$, where

$$
\omega_{\Delta}(x)=\sup _{|t-u| \leq \Delta, 0 \leq t, u \leq T}|x(t)-x(u)| .
$$

Proof of a). Let $\chi^{(m, 1)}(t), \chi^{(m, 2)}(t), \ldots, \chi^{(m, k)}(t), \ldots$ be a sequence of indicator type independent stochastic processes whose finite-dimensional distributions coincide with those of $\chi^{(m)}(t)$. Applying the method of moment generating functions, we conclude that, for a fixed trajectory of the input process $\nu(t)=\left(\nu_{1}(t), \ldots, \nu_{r}(t)\right), t \geq 0$, the distribution of $Q(t)$ coincides with that of

$$
\sum_{m=1}^{r} \sum_{k=1}^{\nu_{m}(t)} \chi^{(m, k)}\left(t-\tau_{k}^{(m)}\right)
$$


E. O. LEBEDEV

This together with equality (1) implies that for $N=1$ the moment generating function $\Phi(t, z), z=\left(z_{1}, \ldots, z_{r}\right),|z| \leq 1$, of the vector $Q(t)$ such that $Q^{\prime}(0)=(0, \ldots, 0)$ can be represented as

$$
\Phi(t, z)=\mathrm{E} \prod_{m=1}^{r} \prod_{k=1}^{\nu_{m}(t)}\left[1-p_{m}^{\prime}\left(t-\tau_{k}^{(m)}\right)(z-\overline{1})\right] .
$$

Consider one-dimensional distributions of the process $\xi^{(n)}(t), t \geq 0$. By

$$
\varphi_{n}(s), \quad s^{\prime}=\left(s_{1}, \ldots, s_{r}\right) \in \mathbf{R}^{r},
$$

we denote the characteristic function of $\xi^{(n)}(t)$. It follows from (3) that

$$
\begin{aligned}
\varphi_{n}(s) & =\mathrm{E} e^{i \xi^{(n) \prime}(t) s} \\
& =\exp \left(-i \sqrt{n} q^{\prime}(t) s\right) \mathrm{E} \exp \left\{\sum_{m=1}^{r} \sum_{k=1}^{\nu_{m}(n t)} \ln \left[1+p_{m}^{\prime}\left(t-\tau_{k}^{(m)} / n\right)\left(e^{i s / \sqrt{n}}-\overline{1}\right)\right]\right\},
\end{aligned}
$$

where

$$
\left(e^{i s / \sqrt{n}}\right)^{\prime}=\left(e^{i s_{1} / \sqrt{n}}, \ldots, e^{i s_{r} / \sqrt{n}}\right) .
$$

Let $\left(s^{2}\right)^{\prime}=\left(s_{1}^{2}, \ldots, s_{r}^{2}\right)$. Then

$$
\begin{aligned}
\lim _{n \rightarrow \infty} \varphi_{n}(s)=\lim _{n \rightarrow \infty} \exp \left(-i \sqrt{n} q^{\prime}(t) s\right) & \\
\quad \times \operatorname{E} \exp \left\{\sum_{m=1}^{r} \sum_{k=1}^{\nu_{m}^{(n)}(n t)}[\right. & \frac{i}{\sqrt{n}} p_{m}^{\prime}\left(t-\frac{\tau_{k}^{(m)}}{n}\right) s-\frac{1}{2} \frac{1}{n} p_{m}^{\prime}\left(t-\frac{\tau_{k}^{(m)}}{n}\right) s^{2} \\
& \left.\left.+\frac{1}{2} \frac{1}{n} s^{\prime} p_{m}\left(t-\frac{\tau_{k}^{(m)}}{n}\right) p_{m}^{\prime}\left(t-\frac{\tau_{k}^{(m)}}{n}\right) s\right]\right\} .
\end{aligned}
$$

Put

$$
W_{k}^{(n)}(t)=\frac{\nu_{k}^{(n)}(n t)-\lambda_{k} n t}{\sqrt{n}}, \quad W^{(n) \prime}(t)=\left(W_{1}^{(n)}(t), \ldots, W_{r}^{(n)}(t)\right) .
$$

The sums on the right-hand side of (4) can be expressed in terms of integrals of $W^{(n)}(t)$ and thus

$$
\begin{aligned}
& \lim _{n \rightarrow \infty} \varphi_{n}(s)= \lim _{n \rightarrow \infty} \exp \left(-i \sqrt{n} q^{\prime}(t) s\right) \\
& \times \operatorname{E} \exp \left\{i \sqrt{n} \lambda^{\prime} \int_{0}^{t} P(u) d u s+i \int_{0}^{t} d W^{(n) \prime}(u) P(t-u)\right. \\
&\left.\quad-\frac{1}{2} \lambda^{\prime} \int_{0}^{t} P(u) d u s^{2}+\frac{1}{2} \sum_{m=1}^{r} \lambda_{m} s^{\prime} \int_{0}^{t} p(u) p^{\prime}(u) d u s\right\} \\
&= \exp \left\{-\frac{1}{2} \sum_{m=1}^{r} \lambda_{m} s^{\prime} \int_{0}^{t}\left[\Delta\left[p_{m}(u)\right]-p_{m}(u) p_{m}^{\prime}(u)\right] d u s\right\} \\
& \times \operatorname{E} \exp \left\{i \int_{0}^{t} d W^{\prime}(u) P(t-u) s\right\} .
\end{aligned}
$$

The right-hand side of the last equality is the characteristic function of

$$
\xi^{(1)}(t)+\xi^{(2)}(t)
$$

The convergence of one-dimensional distributions is proved.

Consider two-dimensional distributions. 
Given a fixed trajectory of the input flow, the distribution of

$$
\left(Q\left(t_{1}\right), Q\left(t_{2}\right)\right), \quad 0<t_{1}<t_{2},
$$

coincides with that of

$$
\sum_{m=1}^{r}\left(\sum_{k=1}^{\nu_{m}\left(t_{1}\right)} \chi^{(m, k)}\left(t_{1}-\tau_{k}^{(m)}\right), \sum_{k=1}^{\nu_{m}\left(t_{1}\right)} \chi^{(m, k)}\left(t_{2}-\tau_{k}^{(m)}\right)+\sum_{k=\nu_{m}\left(t_{1}\right)+1}^{\nu_{m}\left(t_{2}\right)} \chi^{(m, k)}\left(t_{2}-\tau_{k}^{(m)}\right)\right) .
$$

Applying equality (1) for $N=2$ we represent the joint moment generating function $\Phi\left(t_{1}, t_{2}, z(1), z(2)\right)$ of the vectors $Q\left(t_{1}\right), Q\left(t_{2}\right)$ as follows:

$$
\begin{aligned}
\Phi\left(t_{1}, t_{2}, z(1), z(2)\right)=\mathrm{E}\left\{\prod_{m=1}^{r} \prod_{k=1}^{\nu_{m}\left(t_{1}\right)}[1\right. & +p_{m}^{\prime}\left(t_{1}-\tau_{k}^{(m)}\right)(z(1)-\overline{1}) \\
& \left.+p_{m}^{\prime}\left(t_{1}-\tau_{k}^{(m)}\right) \Delta[z(1)] P\left(\Delta t_{2}\right)(z(2)-\overline{1})\right] \\
& \left.\times \prod_{\nu_{m}\left(t_{1}\right)+1}^{\nu_{m}\left(t_{2}\right)}\left[1+p_{m}^{\prime}\left(t_{2}-\tau_{k}^{(m)}\right)(z(2)-\overline{1})\right]\right\} .
\end{aligned}
$$

This representation allows one to evaluate the limit of the joint moment generating function

$$
\varphi_{n}(s(1), s(2)), \quad s(1), s(2) \in \mathbf{R}^{r},
$$

of the vectors $\xi^{(n)}\left(t_{1}\right)$ and $\xi^{(n)}\left(t_{2}\right)$, namely

$$
\begin{aligned}
& \lim _{n \rightarrow \infty} \varphi_{n}(s(1), s(2))=\lim _{n \rightarrow \infty} \mathrm{E} \exp \left(i \xi^{(n) \prime}\left(t_{1}\right) s(1)+i \xi^{(n) \prime}\left(t_{2}\right) s(2)\right) \\
& =\lim _{n \rightarrow \infty} \exp \left(-i \sqrt{n} q^{\prime}\left(t_{1}\right) s(1)-i \sqrt{n} q^{\prime}\left(t_{2}\right) s(2)\right) \\
& \times \mathrm{E}\left\{\sum _ { m = 1 } ^ { r } \left\{\sum _ { k = 1 } ^ { \nu _ { m } ^ { ( n ) } ( n t _ { 1 } ) } \operatorname { l n } \left[1+p_{m}^{\prime}\left(t_{1}-\tau_{k}^{(m)} / n\right)\left(e^{i s(1) / \sqrt{n}}-\overline{1}\right)\right.\right.\right. \\
& +p_{m}^{\prime}\left(t_{1}-\tau_{k}^{(m)} / n\right) \\
& \left.\times \Delta\left[e^{i s(1) / \sqrt{n}}\right] P\left(\Delta t_{2}\right)\left(e^{i s(2) / \sqrt{n}}-\overline{1}\right)\right] \\
& \left.\left.+\sum_{\nu_{m}^{(n)}\left(n t_{1}\right)+1}^{\nu_{m}^{(n)}\left(n t_{2}\right)} \ln \left[1+p_{m}^{\prime}\left(t_{2}-\tau_{k}^{(m)} / n\right)\left(e^{i s(2) / \sqrt{n}}-\overline{1}\right)\right]\right\}\right\} \\
& =\exp \left\{-\frac{1}{2} \sum_{m=1}^{r} \lambda_{m} s^{\prime}(1) \int_{0}^{t_{1}}\left[\Delta\left[p_{m}(u)\right]-p_{m}(u) p_{m}^{\prime}(u)\right] d u s(1)\right. \\
& -\frac{1}{2} \sum_{m=1}^{r} \lambda_{m} s^{\prime}(2) \int_{0}^{t_{2}}\left[\Delta\left[p_{m}(u)\right]-p_{m}(u) p_{m}^{\prime}(u)\right] d u s(2) \\
& \left.-\sum_{m=1}^{r} \lambda_{m} s^{\prime}(1) \int_{0}^{t_{1}}\left[\Delta\left[p_{m}(u)\right]-p_{m}(u) p_{m}^{\prime}(u)\right] d u P\left(\Delta t_{2}\right) s(2)\right\} \\
& \times \mathrm{E}\left\{i \int_{0}^{t_{1}} d W^{\prime}(u) P\left(t_{1}-u\right) s(1)+i \int_{0}^{t_{2}} d W^{\prime}(u) P\left(t_{2}-u\right) s(2)\right\} .
\end{aligned}
$$

The right-hand side of this equality is the characteristic function of the two-dimensional distribution of $\xi^{(1)}(t)+\xi^{(2)}(t)$.

The convergence of $N$-dimensional distributions, $N>2$, can be checked similarly. 
Proof of b). We represent the process $\xi^{(n)}(t)$ as follows:

$$
\xi^{(n)}(t)=\xi^{(1, n)}(t)-\xi^{(2, n)}(t),
$$

where

$$
\begin{gathered}
\xi^{(1, n)}(t)=n^{-1 / 2} \sum_{m=1}^{r}\left(\sum_{k=1}^{\nu_{m}^{(n)}(n t)} p_{m}\left(t-\frac{\tau_{k}^{(m)}}{n}\right)-n \lambda_{m} \int_{0}^{t} p_{m}(t-u) d u\right) \\
\xi^{(2, n)}(t)=n^{-1 / 2} \sum_{m=1}^{r} \sum_{k=1}^{\nu_{m}^{(n)}(n t)}\left[p_{m}\left(t-\frac{\tau_{k}^{(m)}}{n}\right)-\chi^{(m, k)}\left(t-\frac{\tau_{k}^{(m)}}{n}\right)\right] .
\end{gathered}
$$

Since

$$
\omega_{\Delta}\left(\xi^{(n)}\right) \leq \omega_{\Delta}\left(\xi^{(1, n)}\right)+\omega_{\Delta}\left(\xi^{(2, n)}\right),
$$

it is sufficient to check relation (2) for $\xi^{(1, n)}(t)$ and $\xi^{(2, n)}(t)$ separately. We follow the method of the paper [4]. Integrating by parts, we get that for $\Delta>0$,

$$
\Delta \xi^{(1, n) \prime}(t)=\Delta \int_{0}^{t} d W^{(n) \prime}(u) P(t-u)=\Delta W^{(n) \prime}(t)-\int_{-\Delta}^{t} \Delta W^{(n) \prime}(u) d P(t-u),
$$

where

$$
\Delta x(t)=x(t+\Delta)-x(t)
$$

and

$$
W^{(n)}(u)=0
$$

for $u \leq 0$.

Let

$$
\mu_{(r)}=\max _{1 \leq i \leq r} \mu_{i}
$$

Since $P^{\prime}(t)=\Delta(\mu)(P-I) P(t)$,

$$
\sup _{0 \leq t \leq T} \max _{1 \leq i, j \leq r} p_{i, j}^{\prime}(t) \leq \mu_{(r)}
$$

and

$$
\omega_{\Delta}\left(\xi^{(1, n)}\right) \leq\left(1+\mu_{(r)} T\right) \omega_{\Delta}\left(W^{(n)}\right) .
$$

Estimate (5) implies that

$$
\lim _{\Delta \rightarrow 0} \varlimsup_{n \rightarrow \infty} \mathrm{P}\left(\omega_{\Delta}\left(\xi^{(1, n)}\right)>\delta\right)=0
$$

for all $\delta>0$. Now we consider $\xi^{(2, n)}(t), t \in[0, T]$.

The increment $\Delta \xi^{(2, n)}(t)$ can be represented as

$$
\Delta \xi^{(2, n)}(t)=\zeta_{1}+\zeta_{2},
$$


where

$$
\begin{aligned}
\zeta_{1}=n^{-1 / 2} \sum_{m=1}^{r} \sum_{k=1}^{\nu_{m}^{(n)}(n t)} \alpha^{(m, k)}, \quad \zeta_{2}=n^{-1 / 2} \sum_{m=1}^{r} \sum_{\nu_{m}^{(n)}(n t)+1}^{\nu_{m}^{(n)}(n t+n \Delta)} \beta^{(m, k)}, \\
\alpha^{(m, k)}=\left[\chi^{(m, k)}\left(t-\frac{\tau_{k}^{(m)}}{n}\right)-\chi^{(m, k)}\left(t+\Delta-\frac{\tau_{k}^{(m)}}{n}\right)\right] \\
-\left[p_{m}\left(t-\frac{\tau_{k}^{(m)}}{n}\right)-p_{m}\left(t+\Delta-\frac{\tau_{k}^{(m)}}{n}\right)\right] \\
\beta^{(m, k)=} p_{m}\left(t+\Delta-\frac{\tau_{k}^{(m)}}{n}\right)-\chi^{(m, k)}\left(t+\Delta-\frac{\tau_{k}^{(m)}}{n}\right), \\
k=\nu_{m}^{(n)}(n t)+1, \ldots, \nu_{m}^{(n)}(n t+n \Delta) .
\end{aligned}
$$

Let $F_{n}$ be the $\sigma$-algebra generated by the family of random vectors

$$
\left\{\nu^{(n)}(n t), 0 \leq t \leq T\right\} .
$$

Now we obtain an upper bound for $M_{F_{n}}\left(\left|\Delta \xi^{(2, n)}(t)\right|^{4}\right)$ :

$$
\begin{aligned}
& M_{F_{n}}\left(\left|\Delta \xi^{(2, n)}(t)\right|^{4}\right) \leq 8\left(M_{F_{n}}\left|\zeta_{1}\right|^{4}+M_{F_{n}}\left|\zeta_{2}\right|^{4}\right) \\
& \quad \leq 8 r^{4} n^{-2} \sum_{m, i=1}^{r}\left[M_{F_{n}}\left(\sum_{k=1}^{\nu_{m}^{(n)}(n t)} \alpha_{i}^{(m, k)}\right)^{4}+M_{F_{n}}\left(\sum_{\nu_{m}^{(n)}(n t)+1}^{\nu_{m}^{(n)}(n t+n \Delta)} \beta_{i}^{(m, k)}\right)^{4}\right]
\end{aligned}
$$

where $\alpha_{i}^{(m, k)}$ and $\beta_{i}^{(m, k)}$ are the $i$ th coordinates of the vectors $\alpha^{(m, k)}$ and $\beta^{(m, k)}$, respectively. Now we estimate every term in (6) from above.

The random variable

$$
\chi_{i}^{(m, k)}\left(t-\frac{\tau_{k}^{(m)}}{n}\right)-\chi_{i}^{(m, k)}\left(t+\Delta-\frac{\tau_{k}^{(m)}}{n}\right)
$$

assumes only three values $+1,-1$, and 0 , with probabilities

$$
\begin{aligned}
\chi_{i}^{(m, k)}\left(t-\frac{\tau_{k}^{(m)}}{n}\right)-\chi_{i}^{(m, k)}\left(t+\Delta-\frac{\tau_{k}^{(m)}}{n}\right) \\
= \begin{cases}+1, & p_{k}=p_{m i}\left(t-\frac{\tau_{k}^{(m)}}{n}\right)\left(1-p_{i i}(\Delta)\right), \\
-1, & q_{k}=\sum_{j=1, j \neq i}^{r} p_{m j}\left(t-\frac{\tau_{k}^{(m)}}{n}\right) p_{j i}(\Delta), \\
0, & 1-p_{k}-q_{k},\end{cases}
\end{aligned}
$$

respectively. 
It follows from (7) that

$$
\begin{aligned}
n^{-2} M_{F_{n}} & \left(\sum_{k=1}^{\nu_{m}^{(n)}(n t)} \alpha_{i}^{(m, k)}\right)^{4} \leq 3 n^{-2}\left[\sum_{k=1}^{\nu_{m}^{(n)}(n t)}\left(p_{k}+q_{k}\right)+\left(\sum_{k=1}^{\nu_{m}^{(n)}(n t)}\left(p_{k}+q_{k}\right)\right)^{2}\right] \\
\leq & 3 n^{-1} \Delta\left[C_{1}^{(m)}+4 n^{-1 / 2} \mu_{(r)} \sup _{0 \leq t \leq T}\left|W_{m}^{(n)}(t)\right|\right] \\
& +3 \Delta^{2}\left[C_{1}^{(m)}+4 n^{-1 / 2} \mu_{(r)} \sup _{0 \leq t \leq T}\left|W_{m}^{(n)}(t)\right|\right]^{2} \\
= & S_{1, n}^{(m)}(\Delta),
\end{aligned}
$$

where

$$
C_{1}^{(m)}=2 \mu_{(r)} \lambda_{m} \int_{0}^{T}\left(1-p_{m r+1}(u)\right) d u
$$

Similarly we get for the second term on the right-hand side of (6) that

$$
\begin{aligned}
n^{-2} M_{F_{n}} & \left(\sum_{\nu_{m}^{(n)}(n t)+1}^{\nu_{m}^{(n)}(n t+n \Delta)} \beta_{i}^{(m, k)}\right)^{4} \\
\leq & n^{-1}\left[\lambda_{m} \Delta+n^{-1 / 2} \omega_{\Delta}\left(W_{m}^{(n)}\right)+4 \mu_{(r)} n^{-1 / 2} \Delta \sup _{0 \leq t \leq T}\left|W_{m}^{(n)}(t)\right|\right] \\
& +3\left[\lambda_{m} \Delta+n^{-1 / 2} \omega_{\Delta}\left(W_{m}^{(n)}\right)+4 \mu_{(r)} n^{-1 / 2} \Delta \sup _{0 \leq t \leq T}\left|W_{m}^{(n)}(t)\right|\right]^{2} \\
= & S_{2, n}^{(m)}(\Delta) .
\end{aligned}
$$

Combining (8) and (9) we obtain the desired estimate:

$$
M_{F_{n}}\left(\left|\Delta \xi^{(2, n)}(t)\right|^{4}\right) \leq 8 r^{5} \sum_{m=1}^{r}\left(S_{1, n}^{(m)}(\Delta)+S_{2, n}^{(m)}(\Delta)\right),
$$

whence it follows that

$$
\lim _{\Delta \rightarrow 0} \varlimsup_{n \rightarrow \infty} \mathrm{P}\left(\omega_{\Delta}\left(\xi^{(2, n)}\right) \geq 3 \delta\right)=0
$$

for all $\delta>0$. Without loss of generality we assume that $T=1$ and $\Delta=1 / 2^{p}$.

Let

$$
\begin{gathered}
\omega(t, t+\Delta)=\sup _{u \in[t, t+\Delta]}\left|\xi^{(2, n)}(t)-\xi^{(2, n)}(u)\right|, \\
\omega_{\Delta}^{[N]}=\max _{\left|k / 2^{N}-j / 2^{N}\right| \leq \Delta}\left|\xi^{(2, n)}\left(k / 2^{N}\right)-\xi^{(2, n)}\left(j / 2^{N}\right)\right| .
\end{gathered}
$$

Then

for $N>p$, and

$$
\omega_{\Delta}\left(\xi^{(2, n)}\right) \leq \omega_{\Delta}^{[N]}+2 \max _{0 \leq k \leq 2^{N}} \omega\left(\frac{k}{2^{N}}, \frac{k+1}{n}\right)
$$

$$
\mathrm{P}\left(\omega_{\Delta}\left(\xi^{(2, n)}\right) \geq 3 \delta\right) \leq \mathrm{P}\left(\omega_{\Delta}^{[N]} \geq \delta\right)+\mathrm{P}\left(\bigcup_{k=0}^{2^{N}-1}\left\{\omega\left(\frac{k}{2^{N}}, \frac{k+1}{2^{N}}\right) \geq \delta\right\}\right) .
$$

Consider the first term in (11). The random event

$$
\bigcap_{s=p}^{N} \bigcap_{k=1}^{2^{s}}\left\{\left|\xi^{(2, n)}\left(\frac{k}{2^{s}}\right)-\xi^{(2, n)}\left(\frac{k-1}{2^{s}}\right)\right|<\frac{\delta}{s^{2}}\right\}
$$


implies $\left\{\omega_{\Delta}^{[N]}<\delta\right\}$. Passing to the complement events, we get that for $p \geq 3$,

$$
\mathrm{P}\left(\omega_{\Delta}^{[N]} \geq \delta\right) \leq \sum_{s=p}^{N} \sum_{k=1}^{2^{s}} \mathrm{P}\left(\left|\xi^{(2, n)}\left(\frac{k}{2^{s}}\right)-\xi^{(2, n)}\left(\frac{k-1}{2^{s}}\right)\right| \geq \frac{\delta}{s^{2}}\right) .
$$

Using a Chebyshev type inequality for conditional probabilities and estimate (10), we obtain

$$
\begin{aligned}
\lim _{p \rightarrow \infty} & \varlimsup_{n \rightarrow \infty} \mathrm{P}\left(\omega_{\Delta}^{[N]} \geq \delta\right) \\
& \leq \lim _{p \rightarrow \infty} \varlimsup_{n \rightarrow \infty} \sum_{s=p}^{N} \sum_{k=1}^{2^{s}} \mathrm{E}\left\{P_{F_{n}}\left(\left|\xi^{(2, n)}\left(\frac{k}{2^{s}}\right)-\xi^{(2, n)}\left(\frac{k-1}{2^{s}}\right)\right| \geq \frac{\delta}{s^{2}}\right)\right\} \\
& \leq \lim _{p \rightarrow \infty} \varlimsup_{n \rightarrow \infty} \delta^{-4} \sum_{s=p}^{N} s^{8} \sum_{k=1}^{2^{s}} \mathrm{E}\left\{M_{F_{n}}\left|\Delta_{k}^{s} \xi^{(2, n)}\right|^{4}\right\} \\
& \leq 24 \delta^{-4} r^{5} \sum_{m=1}^{r}\left(C_{1}^{(m) 2}+\lambda_{m}^{2}\right) \lim _{p \rightarrow \infty} \sum_{s=p}^{\infty} s^{8} 2^{-s}=0,
\end{aligned}
$$

where

$$
\Delta_{k}^{s} \xi^{(2, n)}=\xi^{(2, n)}\left(\frac{k}{2^{s}}\right)-\xi^{(2, n)}\left(\frac{k-1}{2^{s}}\right)
$$

The equality

$$
\lim _{p \rightarrow \infty} \varlimsup_{n \rightarrow \infty} \sum_{k=0}^{2^{N}-1} \mathrm{P}\left(\omega\left(\frac{k}{2^{N}}, \frac{k+1}{2^{N}}\right) \geq \delta\right)=0
$$

can be checked similarly.

The theorem is proved.

The two terms of the limit process depend on the prelimit processes in the queueing system as follows: $\xi^{(1)}(t)$ is related to the fluctuations of the input flow, while $\xi^{(2)}(t)$ is related to the fluctuations of the service time at the nodes of the network.

\section{Properties of the Limit process}

Prior to our study of the properties of the limit process we give some sufficient conditions for a multidimensional Gaussian process to be Markovian.

Theorem 2. Let $\xi(t)$ be an $r$-dimensional Gaussian process with zero mean vector and such that

a) the correlation functions $R(s)$ and $R(s, t)$ are related by

$$
R(s, t)=R(s) P(t-s), \quad P(t)=\exp (Q t)
$$

for some matrix $Q$ and all $0 \leq s<t$;

b) the matrices $R(s)$ and $R(t)-P^{\prime}(t-s) R(s) P(t-s)$ are nonsingular.

Then the process $\xi(t)$ is Markovian. Moreover the conditional distribution

$$
\mathrm{P}(\xi(t) \in B / \xi(s)=x), \quad B \in B_{R_{r}},
$$

is Gaussian with the mean vector $P^{\prime}(t-s) x$ and correlation matrix

$$
R(t)-P^{\prime}(t-s) R(s) P(t-s) .
$$


The set $G$ of Gaussian processes satisfying condition a) is closed in the sense that if two processes of $G$ are independent and have the same matrix $Q$ in representation a), then every linear combination of them belongs to $G$. As a corollary of Theorem 1 we obtain the following result: the sum of two independent Markov G-processes with the same matrix $Q$ is a Markov process if condition b) holds.

Note that the multidimensional Ornstein-Uhlenbeck process satisfies condition a).

The following result for block matrices is the main tool in the proof of Theorem 1.

Lemma 3. Let $P(t)=\exp (Q t)$ and let $R_{1}, \ldots, R_{n}$ be symmetric $r \times r$ matrices. Assume that

$$
\Delta R_{k+1}=R_{k+1}-P^{\prime}\left(\Delta t_{k+1}\right) R_{k} P\left(\Delta t_{k+1}\right), \quad k=0,1, \ldots, n-1,
$$

are nonsingular, where $0<t_{1}<\cdots<t_{n}, \Delta t_{k+1}=t_{k+1}-t_{k}$, and $R_{0}$ is the zero matrix. Then the block $r n \times r n$ matrix $R$ consisting of $n^{2}$ blocks

$$
R_{i j}= \begin{cases}R_{i} P\left(t_{j}-t_{i}\right), & i \leq j, \\ P^{\prime}\left(t_{i}-t_{j}\right) R_{j}, & i>j,\end{cases}
$$

has the inverse matrix $R^{-1}=\left\|R_{i j}^{(-1)}\right\|_{1}^{n}$, which is three-diagonal. Moreover,

$$
\begin{gathered}
R_{i i-1}^{(-1)}=-\Delta R_{i}^{(-1)} P^{\prime}\left(\Delta t_{i}\right), \quad i=2, \ldots, n, \\
R_{i i+1}^{(-1)}=-P\left(\Delta t_{i+1}\right) \Delta R_{i+1}^{(-1)}, \quad i=1, \ldots, n-1, \\
R_{i i}^{(-1)}=\Delta R_{i}^{-1}+P\left(\Delta t_{i+1}\right) \Delta R_{i+1}^{-1} P^{\prime}\left(\Delta t_{i+1}\right), \quad i=1, \ldots, n-1, \quad R_{n n}^{(-1)}=\Delta R_{n}^{-1} .
\end{gathered}
$$

To prove Lemma 3 we use induction on $n$ and the following result: if a square matrix $A$ is of the block form

$$
A=\left(\begin{array}{ll}
A_{11} & A_{12} \\
A_{21} & A_{22}
\end{array}\right),
$$

where $A_{11}$ and $A_{22}$ are square matrices, then $A^{-1}$ is also a block matrix, and moreover,

$$
A^{-1}=\left(\begin{array}{ll}
A_{11}^{(-1)} & A_{12}^{(-1)} \\
A_{21}^{(-1)} & A_{22}^{(-1)}
\end{array}\right),
$$

where

$$
\begin{gathered}
A_{22}^{(-1)}=\left[A_{22}-A_{21} A_{11}^{-1} A_{12}\right]^{-1}, \quad A_{11}^{(-1)}=A_{11}^{-1}+A_{11}^{-1} A_{12} A_{22}^{(-1)} A_{21} A_{11}^{(-1)}, \\
A_{21}^{(-1)}=-A_{22}^{(-1)} A_{21} A_{11}^{(-1)}, \quad A_{12}^{(-1)}=-A_{11}^{-1} A_{12} A_{22}^{(-1)},
\end{gathered}
$$

provided the inverse matrices on the right-hand side of (12) exist. A similar result can be found in [5].

The proof of Theorem 2 follows from Lemma 1 and Theorem 2 in [3], p. 262 .

It is clear that the limit $\xi^{(1)}(t), t \geq 0$, is an $r$-dimensional Ornstein-Uhlenbeck process. The following is an immediate corollary of Theorem 2 for the sum $\xi^{(1)}(t)+\xi^{(2)}(t)$.

Corollary 1. If the spectral radius of the matrix $P$ does not exceed 1 , then the limit Gaussian process $\xi^{(1)}(t)+\xi^{(2)}(t)$ is an $r$-dimensional diffusion process with the shift vector $A(x)=Q^{\prime} x$ and diffusion matrix

$$
B(t)=\Delta\left[q^{\prime}(t) Q\right]-Q^{\prime} \Delta[q(t)]-\Delta[q(t)] Q+\sigma^{2},
$$

where $Q=\Delta(\mu)(P-I)$ and $\Delta(x)$ is a diagonal matrix whose principal diagonal coincides with the vector $x$.

Theorem 1 is a result of the diffusion approximation type. Note also that Theorem 1 contains more information about the structure of the limit process than do other results of this type. 


\section{An APPLiCATion FOR NETWORKS With A SEMI-MARKOV INPUT}

Consider a particular case of a $[G|M| \infty]^{r}$ network where the input flow has a special structure. We assume that $r$ servers have a common input flow of customers governed by a semi-Markov process $\zeta(t) \in\{1,2, \ldots, N\}$. This means that the arrival times of customers coincide with the moments $\tau_{n}, n=1,2, \ldots$, at which the process $\zeta(t)$ changes its state. If the process $\zeta(t)$ moves to a state " $i$ " at a moment $\tau_{n}$, then the probability that the $n$th customer arrives at the server $j$ is $h_{i j}, \sum_{j=1}^{r} h_{i j}=1$. The matrix $H=\left\|h_{i j}\right\|$ is of size $N \times r$. Denote by

$$
F(t)=\left\|F_{i j}(t)\right\|_{1}^{N}
$$

the semi-Markov matrix of the process $\zeta(t)$. Let

$$
F_{i}(t)=\sum_{j=1}^{N} F_{i j}(t)
$$

be the distribution function of the time spent by the process $\zeta(t)$ at the state " $i$ ", let

$$
f_{i j}=F_{i j}(+\infty)
$$

be the transient probabilities of the embedded Markov chain, and $F=\left\|f_{i j}\right\|_{1}^{N}$. Such a multichannel network with the input flow specified above is denoted by $[S M|M| \infty]^{r}$ in the theory of queues.

It is known that condition 1 ) of Theorem 1 holds for the input flows $\nu_{1}(t), \ldots, \nu_{r}(t)$ if

3) the matrix $F$ is indecomposable;

4) there exist the first and second moments of the time spent at every state,

$$
m_{i}=\int_{0}^{\infty} t d F_{i}(t)<\infty, \quad d_{i}=\int_{0}^{\infty} t^{2} d F_{i}(t)<\infty, \quad i=1,2, \ldots, N
$$

(see 6, 7]).

Following the method of the paper [8], we represent the intensities $\lambda_{i}, i=1, \ldots, r$, and the matrix $\sigma^{2}$ as follows:

$$
\begin{gathered}
\lambda_{i}=\frac{1}{m} \sum_{j=1}^{N} \pi_{j} h_{j i}, \quad i=1, \ldots, r, \\
\sigma^{2}=H^{\prime} C H+\frac{1}{m} \sum_{j=1}^{N} \pi_{j}\left[\Delta\left(h_{j}\right)-h_{j} h_{j}^{\prime}\right], \\
C=\left\|c_{\alpha \beta}\right\|_{1}^{N}, \\
c_{\alpha \beta}=\pi_{\alpha} \frac{1}{m} \sum_{j=1}^{N} r_{\alpha j} f_{j \beta}+\pi_{\beta} \frac{1}{m} \sum_{j=1}^{N} r_{\beta j} f_{j \alpha}+\pi_{\alpha} \pi_{\beta} \frac{d-2 m^{(2)}}{m^{3}}+\delta_{\alpha \beta} \frac{\pi_{\alpha}}{m}, \\
R_{1}=\left\|r_{i j}\right\|_{1}^{N}=\left(I-\frac{1}{m} \Pi \Delta(m)\right) R_{0}\left(I-\frac{1}{m} \Delta(m) \Pi\right) .
\end{gathered}
$$

Here $\pi_{1}, \pi_{2}, \ldots, \pi_{N}$ and $h_{j}^{\prime}=\left(h_{j 1}, \ldots, h_{j r}\right)$ are the stationary distribution of the embedded chain and the $j$ th row of the matrix $H$, respectively,

$$
m=\sum_{i=1}^{N} m_{i} \pi_{i}, \quad d=\sum_{i=1}^{N} d_{i} \pi_{i}, \quad m^{(2)}=\sum_{i=1}^{N} m_{i}^{2} \pi_{i},
$$

$\Delta(m)=\left\|m_{i} \delta_{i j}\right\|_{1}^{N}, \Pi$ is an $N \times N$ matrix whose rows are equal to each other and coincide with the stationary distribution, and $R_{0}=(I-F+\Pi)^{-1}-\Pi$ is the potential of the embedded Markov chain. 
The following result is a corollary of Theorem 1 .

Theorem 3. Assume that conditions 2)-4) hold for a queueing $\left[S M^{(n)}\left|M^{(n)}\right| \infty\right]^{r}$ network and the spectral radius of the route matrix $P$ is less than 1 . Then the normalized queueing process $\xi^{(n)}(t)$ weakly converges in the uniform topology on every finite interval $[0, T]$ to a diffusion process $\xi(t)(\xi(0)=0)$ with the shift vector $A(x)=Q^{\prime} x$ and diffusion matrix

$$
B(t)=\Delta\left[q^{\prime}(t) Q\right]-Q^{\prime} \Delta[q(t)]-\Delta[q(t)] Q+\sigma^{2},
$$

where the matrix $\sigma^{2}$ is defined by (13)-(15).

The convergence of the functionals of the process $\xi^{(n)}(t)$ can be used to evaluate the quality index of a network and the optimal control for the service processes.

\section{BIBLIOGRAPHY}

1. W. A. Massey and W. Whitt, A stochastic model to capture space and time dynamics in wireless communication systems, Probability in the Engineering and Informational Sciences 8 (1994), 541-569.

2. A. Dvurechenskiŭ, L. A. Kulyukina, and G. A. Ososkov, Estimates of the Primary Ionization in Ionization Chambers, Preprint 5-81-362, Joint Institute for Nuclear Research, Dubna, 1981. (Russian)

3. I. I. Gikhman, A. V. Skorokhod, and M. I. Yadrenko, Probability Theory and Mathematical Statistics, "Vyshcha Shkola", Kiev, 1988. (Russian)

4. A. A. Borovkov, Asymptotic Methods in the Queueing Theory, "Nauka", Moscow, 1980; English transl., Wiley, New York, 1984.

5. R. A. Horn and C. R. Johnson, Matrix Analysis, Cambridge University Press, Cambridge, 1985. MR 87e:15001

6. V. V. Anisimov, Limit theorems for semi-Markov processes with a finite phase space, Dokl. Akad. Nauk SSSR 193 (1970), no. 3, 503-505; English transl. in Soviet Math. Dokl. 11 (1970), 945-948. MR 42:3851

7. D. S. Silvestrov, Limit theorems for functionals of step processes constructed from sums of random variables defined on a semi-Markov process with a finite phase space, Dokl. Akad. Nauk SSSR 195 (1970), no. 5, 1036-1038; English transl. in Soviet Math. Dokl. 11 (1971). MR $42: 8547$

8. V. S. Korolyuk and A. F. Turbin, Semi-Markov Processes and Their Applications, "Naukova Dumka", Kiev, 1976. (Russian) MR 54:8913

Department of Applied Statistics, Faculty for Cybernetics, Kyiv National Taras Shevchenko University, Academician Glushkov Avenue 4, Kyiv-127 03127, Ukraine

E-mail address: leb@unicyb.kiev.ua

Received 10/DEC/2001

Translated by V. SEMENOV 